\title{
Effect of Halogen Substitution on the Absorption and Emission Profile of Organometallic Perovskites
}

\author{
Ling Jin Kiong ${ }^{1}$, and Jose Rajan ${ }^{1}$ \\ ${ }^{1}$ University Malaysia Pahang, Faculty of Industrial Sciences \& Technology, Material Technology \\ Department, Advance Material Laboratory, Lebuhraya Tun Razak, 26300 Gambang, Kuantan, \\ Pahang Darul Makmur.
}

\begin{abstract}
Comparative study on the optical properties of methylamide lead mono- and hybrid-halide perovskite samples, from the perspective of its crystal structure, which were synthesized in ambient condition (temperature $\sim 26.6{ }^{\circ} \mathrm{C}$, humidity $\sim 65 \%$ ), a step toward industrial commercialization, were carried out. In this study, in-depth information on both crystal structure and optical properties of the methylamide lead halide perovskite was the main focus, including study of relationship between these two factors via real world experimentation. Increment in lattice stain can be observed when the bromide or chloride ions were substituted, resulting in higher lattice strain in hybrid-halide samples. The lattice strain provides kinetic energy to the electrons, facilitating the promotion of exciton which increased the number of photon emitted during recombination. However, increasing lattice strain results in the widening of the energy gap of the samples by shifting the energy level to a higher or lower level. The results provide some insight on the relationship between crystal structure and optical properties of perovskite which paces a route for future cross-factor experimentation under uncontrolled ambient environment, in an effort to unravel more underlying working mechanism of perovskite solar cells.
\end{abstract}

\section{Introduction}

In response to Paris Agreement, more than 120 countries had put in effort to counteract climate changes. One of their efforts is by implementing conversion of traditional industries to green industries, which result in advancement of photovoltaic industries all around the globe [1]. To ensure the further development of photovoltaic (PV) industry, improvement of $\mathrm{PV}$ efficiencies as well as performance and reduction in product's cost are both important. Perovskite solar cells (PSCs) were first introduced in 2009, with its efficiency leapfrogged from $\sim 3.8 \%$ [2] to $\sim 21 \%$ [3] in 2016, had attracted attention from the research community. Its capability of optimal operation even in low light intensity and solution-processability, which offer low cost fabrication, make PSCs a suitable candidate to replace non-economical silicon solar cells (SSCs) that monopolized nowadays photovoltaic industry. In addition, both nano-scale thickness and absence of liquid electrolyte opened up huge gateway for the application of PSCs technology, such as building integrated photovoltaic system (BIPV) or 
flexible photovoltaic device. Aside from pros, the biggest con of PSCs will be its stability. Efforts had been taken to improve the stability of PSCs, including introducing a polymeric framework to the perovskite crystal structure to prolong the decomposition of the perovskite film but results in poor fill factor $(<40 \%)$ and large hysteresis $(\sim 50 \%)$ [4]. A study done by Fakharuddin et. al. had reported that not only the perovskite film itself but the morphology, porosity and chemical stability of the electron transport layer play a vital role as well in determining the stability of PSCs where the application of $\mathrm{TiO} 2$ nanorod instead of conventional $\mathrm{TiO} 2$ layer had demonstrated higher PSCs durability [5]. Though glance on some of the factors affecting the stability had been obtained, ways of improving the stability without affecting the PSCs performance had not yet been identified. Fabrication cost of PSCs, been the main factor determining PSCs marketability, had been revised to reduce the overall cost of PSCs manufacturing by remodeling the fabrication method, from one-step deposition method to two-step deposition method, as well as from solution deposition to evaporation techniques [6-9]. Studies on the underlying working mechanism of PSCs had also been carried out as an effort to understand the physics of PSCs and to gain new insight for further PSCs device improvement. Stranks et. al. had reported that the incorporation of chloride atoms into the MAPbI3 structure will helps to improve the diffusion length of electrons and reduces the recombination rate with no focus on the optoelectronic properties [10]. The effect of non-stoichiometric precursors on MAPbI3 PSCs had also been investigated, showing an improvement on the photovoltaic performance [11] as well as the fill factor [12] in step with the increasing PbI2 amount. Instead of real-life experiment, several studies are done using computer simulation, mostly using Density Functional Theory (DFT), MD simulation, Group theory and GW approximation, to unravel the underlying working mechanism and physics of the ABX3 perovskite material [13-16].

Computation simulation was able to estimate and explain the physics and working mechanism observed from PSCs devices under specific set of condition, which might be totally different in real-life ever-changing environmental condition. On the other hand, the stability of perovskite film cannot be studied using computer simulation method, indicating the importance of real-time experimentation in further perovskite investigation. With both real-time experimentations and computational simulations done by previous researchers, it is undeniable that adequate knowledge about perovskite had been revealed despite from some yet unraveled secret hidden within the physics of perovskite. Almost all the previous studies are carried out in line with 3 main directions, which are (i) improving perovskite stability, (ii) improving efficiency and performance of PSCs, and (iii) studying the physics of perovskite. Seldom a cross direction study is been carried out before, which might offer some recommendation on ways for PSCs improvement. One-directional study does provide some explanation on PSCs performance but seldom provide in-depth information regarding the physics of PSCs and the overall relationship between the crystal structure of perovskite and its optoelectronic properties. Therefore, a cross-directional study is crucial for PSCs to breakthrough from it efficiency bottleneck.

Herein, we studied the optical properties of perovskite from the perspective of crystal structure when the halide of $\mathrm{MAPbX} 3(\mathrm{X}=\mathrm{I}, \mathrm{Br}$, or $\mathrm{Cl})$ is substituted. A total of 9 samples are studied, including 5 mono-halide MAPbI3 samples with different molar ratio and 4 hybrid-halide MAPbI3-xYx samples (where $\mathrm{Y}=\mathrm{Br}$ or $\mathrm{Cl}$; $\mathrm{X}=1$ or 2). One-step solutiondecomposition technique is used to prepare all 9 samples under ambient environment (temperature $\sim 26.6^{\circ} \mathrm{C}$, humidity $\sim 65 \%$ ), which is one of the crucial factor in future low cost industrial commercialization. It is well known that the optoelectronic properties of perovskite originated from its non-stoichiometry crystal structure. Our main focus is to study the effect of halide substitution on perovskite crystal structure and its effect on perovskite optical properties from the crystal structure point of view. The crystal structure of the perovskite samples were studied using X-ray Diffraction (XRD) and its optical properties were obtained 
using UV-Vis-NIR Absorption Spectroscopy as well as Photoluminescence Spectroscopy. We reported a detailed relationship between crystal structure and optical properties of perovskite samples when the halide, $\mathrm{X}$ of MAPbX3 is substituted.

\section{Experimental Section}

\subsection{Perovskite Preparation}

One-step solution deposition technique was adopted in this study to synthesis organometallic halide perovskite. All the chemicals used in this study were obtained from Aldrich-sigma and used without any further purification. Methylamine $\left(\mathrm{CH}_{3} \mathrm{NH}_{3}, 33 \mathrm{wt} \%\right.$ in absolute ethanol) was mixed with hydroiodic acid ( $\mathrm{HI}, 57 \mathrm{wt} \%$ in $\mathrm{H}_{2} \mathrm{O}$ ) at $0{ }^{\circ} \mathrm{C}$ by stirring for 2 hours, followed by evaporation using rotatory evaporator at $70{ }^{\circ} \mathrm{C}$ for 1 hour. The precipitate was then cleaned using diethyl ether for three times until a white color precipitate was obtained followed by drying in oven at $70{ }^{\circ} \mathrm{C}$ overnight. The methylamide iodide, MAI powder synthesized was used for the following sample preparation without any further purification. Lead (II) iodide $\left(\mathrm{PbI}_{2}, 99 \%\right)$ powder and the prepared methylamide iodide powder were mixed, $40 \mathrm{wt} \%$ in dimethylformamide, DMF solution $\left(\mathrm{EMSURE}^{\circledR}\right.$ ) in the ratio of $1: 3 ; 1: 2 ; 1: 1 ; 1: 2 ;$ and $1: 3$. The resultant solutions were stirred at $70{ }^{\circ} \mathrm{C}$ overnight. Hybrid-halide perovskite samples were prepared by mixing the above prepared MAI powder with lead (II) bromide $\left(\mathrm{PbBr}_{2}\right.$, 99\%) powder or lead (II) chloride $\left(\mathrm{PbCl}_{2}, 99 \%\right)$ powder, $40 \mathrm{wt} \%$ in DMF solution, followed by stirring at $70{ }^{\circ} \mathrm{C}$ for overnight. Ratio of (MAI:PbI2:PbX2) used in hybrid-halide perovskite preparation were $(1: 0.33: 0.67)$ and (1:0.67:0.33). For perovskite film preparation, titanium dioxide, $\mathrm{TiO}_{2}$ paste (Solaronix) was dissolved in ethanol with a weight ratio of $1 \mathrm{wt} \%$ of $\mathrm{TiO}_{2}$ paste in $6 \mathrm{wt} \%$ of ethanol. The $\mathrm{TiO}_{2}$ electron transport layer was then deposited on glass slide by spin-coating at $1000 \mathrm{rpm}$ for 20 seconds. The substrates were heated at $500{ }^{\circ} \mathrm{C}, \sim 1$ hour for complete drying. Once the substrate cooled down, perovskite solution was deposited via spin-coating at $1000 \mathrm{rpm}$ for 15 seconds, followed by substrate heating at $70{ }^{\circ} \mathrm{C}$ for 1 hour. A dark colored perovskite film was obtained for all the sample.

\subsection{Sample Characterizations}

Absorption profile of the samples were recorded using UV-Vis-NIR absorption spectroscopy, UV-2600 of Shimadzu with integrated sphere 2600 plus. Single scan mode was applied, with medium scanning speed, scanning from $900 \mathrm{~nm}$ to $400 \mathrm{~nm}$, peak's threshold value set to be 0.001 at points 4 and slit width of $5 \mathrm{~nm}$. The light source changed wavelength at $323 \mathrm{~nm}$ whereas the detector change wavelength at $830 \mathrm{~nm}$. The emission profiles of all the samples were recorded using photoluminescence spectroscopy, Edinburgh Instruments NIR 300/2. Source light path used is Xe900 with wavelength $300 \mathrm{~nm}$ whereas the emission detector light path used was Visible $500 \mathrm{~nm}$ with iris set at 95, scanning wavelength ranged from $620 \mathrm{~nm}$ to $900 \mathrm{~nm}$ with excitation wavelength set at $600 \mathrm{~nm}$, bandwidth of $20 \mathrm{~nm}$, and $0.5 \mathrm{~s}$ dwell time. To study the crystal structure of the samples, X-ray diffraction Bruker, D8 Advance model was employed. The XRD spectra were taken within the range of 5 to $60^{\circ} 2 \boldsymbol{\theta}$ at room temperature using $\mathrm{CuK} \alpha$ radiation. Tube voltage was adjusted to be $40 \mathrm{kV}$, tube current of $40 \mathrm{~mA}$, step-scan mode with a step size of $0.02^{\circ} 2 \boldsymbol{\theta}$ and counting step of $1-3 \mathrm{~s}$ per step. 


\section{RESULTS AND DISCUSSION}

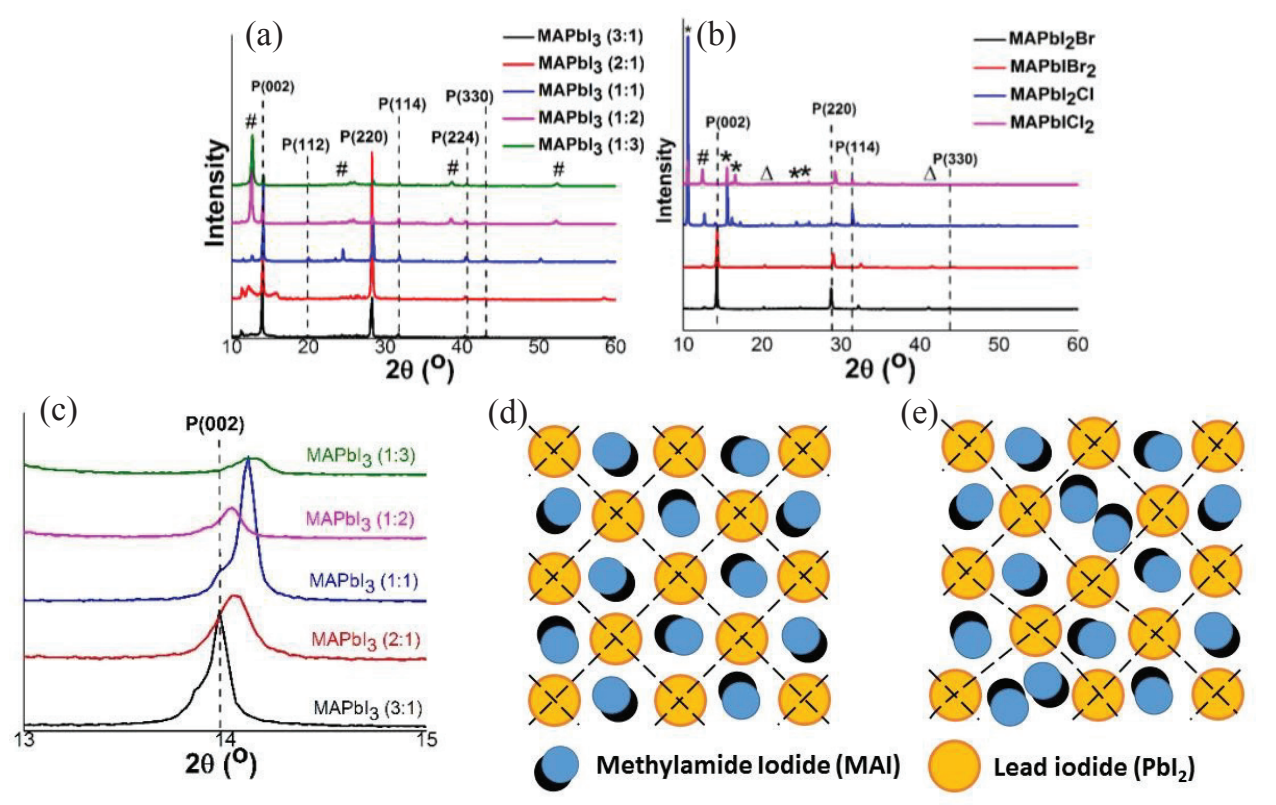

Figure 1: XRD spectra for mono-halide sample (a) and hybrid-halide samples (b), indicating peaks for perovskite (P), $\mathrm{PbI}_{2}(\#), \mathrm{PbBr}_{2}(\Delta)$, and $\mathrm{PbCl}_{2}(*)$. Graph (c) highlighted the left-skewed behavior of the perovskite (002) crystal plane when the MAI:PbI 2 ratio was altered. (d) and (e) illustrates the lattice strain experienced by the crystal structure when amount of MAI molecules increases.

Briefly, fig 1(a) and 1(b) show the XRD spectra of all the samples. All 9 samples show tetragonal crystal structure with lattice parameter $\mathrm{a}=\mathrm{b}=\sim 8.9 \AA, \mathrm{c}=\sim 12.6 \AA$ for $\mathrm{MAPbI}_{3}$ as well as $\mathrm{MAPbI}_{3-\mathrm{x}} \mathrm{Cl}_{\mathrm{x}}$ samples, and $\mathrm{a}=\mathrm{b}=\sim 8.7 \AA, \mathrm{c}=\sim 12.4 \AA$ for $\mathrm{MAPbI}_{3-\mathrm{x}} \mathrm{Br}_{\mathrm{x}}$ samples respectively. Regardless to mono-halide or hybrid-halide perovskite samples, all the samples shown high peak intensity at $\sim 14^{\circ}$, indicating plane (002) as the main crystal plane in all the perovskite samples, excluding mono-halide sample with ratio $(2: 1)$ where the main crystal plane was plane (220). By studying the position of the (002) crystal plane of mono-halide perovskite samples, the peak of the (002) planes were clearly left-skewed when the amount of MAI added was increased, as illustrated in fig 1(c). To study this left-skewed behavior of the (002) plane when MAI amount was altered, Williamson-Hall equation was applied to calculated the lattice strain and average crystallite size of the samples.

$$
\begin{gathered}
B_{t} \cos \theta=\frac{k \lambda}{L}+4 \varepsilon \cos \theta \\
B_{t}^{2}=B_{\text {measure }}^{2}+B_{\text {Instrumental }}^{2}
\end{gathered}
$$

$\mathrm{B}_{\mathrm{t}}$ represent the sample line broadening, calculated using equation $2, \mathrm{k}$ is the crystal constant, $\sim 0.94, \lambda$ is the X-ray wavelength used in XRD testing, $\mathrm{L}$ is the average crystallite size, $\boldsymbol{\varepsilon}$ is the lattice strain, $\boldsymbol{\theta}$ represent the angle, $\mathrm{B}_{\text {measure }}$ represent the peak broadening at FWHD, and $\mathrm{B}_{\text {Instrumental }}$ is the line broadening results from the measuring instrument which can be obtained from the peak of the standard sample. 
Table 1: Lattice parameter, lattice strain, average crystallite size of the perovskite samples.

\begin{tabular}{|c|c|c|c|c|}
\hline \multirow[b]{2}{*}{ Sample } & \multicolumn{2}{|c|}{ Lattice parameter $(\AA)$} & \multirow[b]{2}{*}{ Lattice strain } & \multirow{2}{*}{$\begin{array}{c}\text { Average } \\
\text { crystallite size } \\
(\mathrm{nm})\end{array}$} \\
\hline & $\mathbf{a}=\mathbf{b}$ & c & & \\
\hline $\mathrm{MAPbI}_{3}(3: 1)$ & 8.9644 & 12.6764 & 0.0312 & 115.206 \\
\hline $\mathrm{MAPbI}_{3}(2: 1)$ & 8.9097 & 12.6213 & 0.0091 & 448.697 \\
\hline $\mathrm{MAPbI}_{3}(1: 1)$ & 8.8826 & 12.5484 & 0.0080 & 435.742 \\
\hline $\mathrm{MAPbI}_{3}(1: 2)$ & 8.9187 & 12.6212 & 0.0103 & 335.776 \\
\hline $\mathrm{MAPbI}_{3}(1: 3)$ & 8.8646 & 12.5122 & 0.0064 & 497.908 \\
\hline $\mathrm{MAPbI}_{2} \mathrm{Br}$ & 8.7758 & 12.4229 & 0.0103 & 337.370 \\
\hline $\mathrm{MAPbIBr}_{2}$ & 8.7234 & 12.3875 & 0.0096 & 354.750 \\
\hline $\mathrm{MAPbI}_{2} \mathrm{Cl}$ & 8.9644 & 12.5665 & 0.0250 & 142.675 \\
\hline $\mathrm{MAPbICl}_{2}$ & 8.9828 & 12.6212 & 0.0107 & 332.705 \\
\hline
\end{tabular}

From table 1, it was noticeable that the lattice strain experienced by $(3: 1)$ ratio sample was higher compared to the equimolar ratio sample. The higher lattice strain experienced by (3:1) ratio sample originated from the vacancy occupation of excess MAI molecules, illustrated in figure 1(e). When excess MAI were mixed to the PbI2 powder, the cation A site will be occupied by more than one MAI molecules, stretching the crystal structure, creating some degree of strain on the crystal structure. The strain increased when more number of MAI molecules occupied the cation A site, stretching the crystal lattice. Besides, the peak for $\mathrm{PbI} 2$ hexagonal crystal structure can be observed on perovskite sample with ratio (1:2) and (1:3). The insufficient amount of MAI molecules to occupy the cation A site resulted in empty cation A site, which increased the tendency for the PbI2 tetragonal cage to form pure $\mathrm{PbI} 2$ hexagonal crystal structure. The peak intensity for $\mathrm{PbI} 2$ plane, located at $\sim 12.6^{\circ}$, increased when the amount of PbI2 in the perovskite sample increased, as shown in the XRD spectra for both sample with ratio (1:2) and (1:3) respectively. No PbI2 hexagonal crystal structure peak can be observed in the $(3: 1)$ perovskite sample where all the cation A sites were occupied by the over sufficient MAI molecules, and therefore reducing the convertion tendency from $\mathrm{PbI} 2$ tetragonal cage to hexagonal crystal structure. The PbI2 hexagonal crystal structure peak can also be detected by XRD from hydrated perovskite structure upon longer exposure duration to the ambient environment. In this study, the XRD spectra were obtained instantaneously after the samples were prepared. Therefore, the possibility that the $\mathrm{PbI} 2$ hexagonal crystal structure peak originated from a hydrated perovskite sample was very insignificant and can be ruled out. Comparison in between hybrid-halide samples highlighted higher lattice strain experienced by the chloride-substituted sample than the bromidesubstituted sample. Chloride ions, with a smaller ionic radii compared to the bromide, created more vacancy when substituting iodide ions, causing the crystal space to shrink in order to reduce the vacancy space, generating more strain on the crystal lattice compared to bromide ions. However, when the amount of substitution ions, such as bromide and chloride, increases, the lattice strain experienced by the crystal structure reduced. In terms of lattice parameters, the bromide-substituted samples had a smaller value of lattice parameters, due to the bromide's smaller ionic radii. However, that was not the case of chloride -substituted samples. In spite of smaller ionic radii, the lattice parameter of the chloride-substituted samples were almost the same as the mono-halide samples. The radius of chloride ions might be too small to have any effect on the overall perovskite crystal parameter. With the study done on the effect of halogen substitution on the crystal structure of the perovskite, changed on the optical profile of the perovskite can be studied in the perspective of perovskites' crystal structure. 

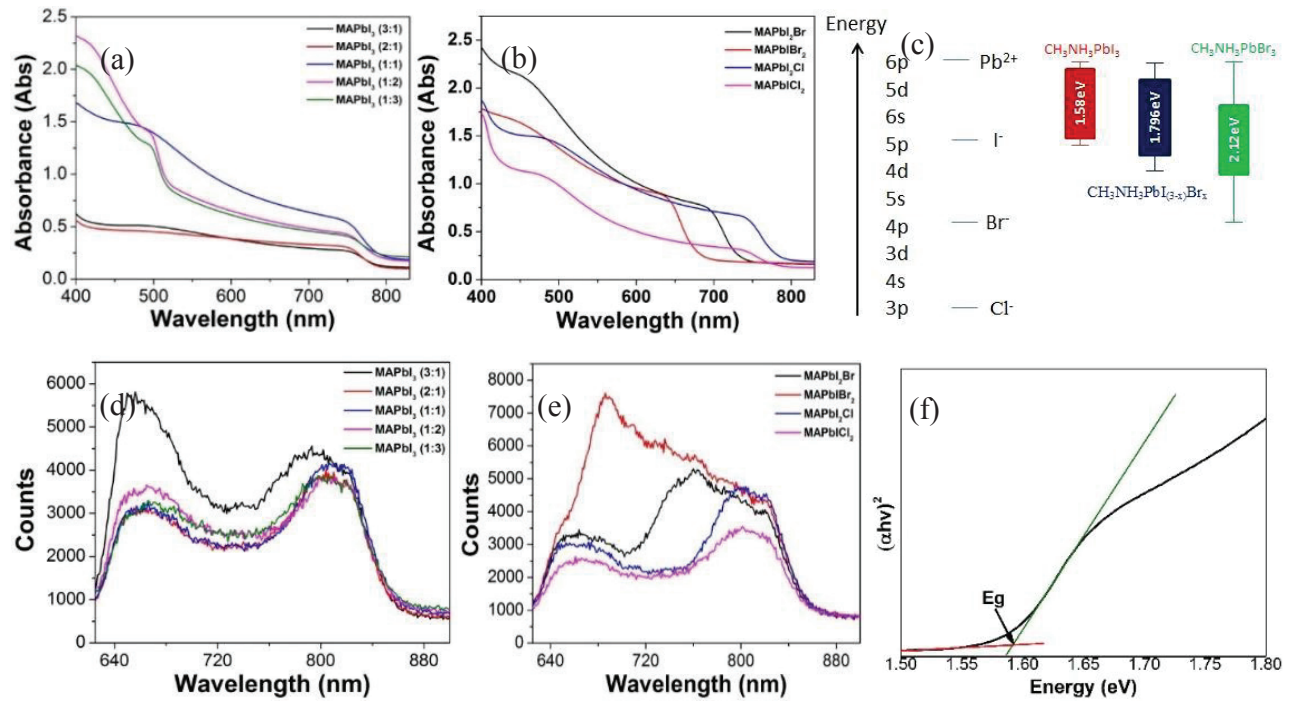

Figure 2: (a) and (b) illustrated the absorption spectra for mono-halide and hybrid-halide samples respectively, (c) illustrated the energy diagram of the mono-halide and hybrid-halide samples, (d) and (e) illustrated the photoemission spectra for mono-halide and hybrid-halide samples respectively, lastly (f) energy gap investigation using Tauc's plot technique.

Table 2: The calculated tolerance factor and energy gap calculated using Tauc's plot technique.

\begin{tabular}{cccccc}
\hline No. & Sample & $\begin{array}{c}\text { Tolerance } \\
\text { Factor }(\mathbf{t})\end{array}$ & $\begin{array}{c}\text { Energy } \\
\text { gap }(\mathbf{e V})\end{array}$ & $\begin{array}{c}\text { Absorption } \\
\text { cross section }\end{array}$ & $\begin{array}{c}\text { Photoemission } \\
\text { cross section }\left(\mathbf{1 0}^{5}\right)\end{array}$ \\
\hline 1. & $\mathrm{MAPbI}_{3}(3: 1)$ & 0.9175 & 1.598 & 166.1 & 9.03 \\
2. & $\mathrm{MAPbI}_{3}(2: 1)$ & 0.9175 & 1.582 & 162.2 & 6.52 \\
3. & $\mathrm{MAPbI}_{3}(1: 1)$ & 0.9175 & 1.575 & 405.5 & 6.74 \\
4. & $\mathrm{MAPbI}_{3}(1: 2)$ & 0.9175 & 1.566 & 378.9 & 7.05 \\
5. & $\mathrm{MAPbI}_{3}(1: 3)$ & 0.9175 & 1.557 & 352.9 & 6.94 \\
6. & $\mathrm{MAPb}_{2} \mathrm{Br}$ & 0.9233 & 1.710 & 470.5 & 8.56 \\
7. & $\mathrm{MAPb}_{2}$ & 0.9294 & 1.849 & 373.6 & 11.60 \\
8. & $\mathrm{MAPbI}_{2} \mathrm{Cl}$ & 0.9271 & 1.597 & 421.9 & 7.20 \\
9. & $\mathrm{MAPbICl}_{2}$ & 0.9376 & 1.590 & 268.7 & 5.97 \\
\hline
\end{tabular}

Figure 2 (a), (b), (d) and (e) illustrated the absorption and photoemission spectra for mono-halide and hybrid halide perovskite respectively. The energy gap of the perovskite materials were obtained using the Tauc's plot method where the absorption coefficient, $\alpha$ of the materials were calculated using equation 3.

$$
\alpha=\left(\frac{1}{t}\right)\left(\frac{A}{\log e}\right)
$$

Where $t$ represent the thickness of the sample, $\mathrm{A}$ is the absorbance of the material, and e is the electron charge $\left(\mathrm{e}=1.6 \times 10^{-19} \mathrm{C}\right)$. Graph of $(\alpha \mathrm{hv})^{2}$ against hv was plotted, as shown in fig 2(f), where the intersection point of the two tangent point was the energy gap of the studied materials. The calculated energy gaps were recorded in table 2 where clear observation can be made where the energy gap widened when the amount of cation A added increases. Additional amount of cation A might played an important part in altering the total number of charge within the perovskite crystal structure, where different amount of free charges will altered the level of the Fermi energy. However, the electric field in perovskite samples were not generated by the free electrons, but from the overall charge of the perovskite structure due to the position orientation of the cation A. The lattice strain 
experienced by the crystal lattice do have some degree of influence on the Fermi energy level, but the small lattice strain experienced by the crystal structure had no significant effect on the Fermi energy level in this case. From Schrondinger equation, illustrated in equation 4, the different in charges might affect the conduction level as well.

$$
\begin{gathered}
n=2\left(\frac{2 \pi m_{e}^{*} k T}{h^{2}}\right)^{3 / 2} \exp \left(\frac{E_{F}-E_{C}}{k T}\right) \\
E_{F 1}-E_{C 1}=\log \left(\frac{n_{1}}{n_{3}}\right)\left(E_{F 2}-E_{C 2}\right)
\end{gathered}
$$

Equation 4 can be further derived into eq 5, explaining the relationship between different number of charges on the energy level of Fermi energy and conduction band energy level of two different samples. In this study, since the different in Fermi energy level due to the lattice strain was very insignificant, conduction energy level will be the main focus. The additional MAI molecules in cation A site might reduce the overall charge of the perovskite crystal by forming secondary bonding with the surrounding $\mathrm{PbI}_{2}$ tetragonal cage, which were previously left unbounded. From eq 5, less charge will eventually widened, or increased, the energy level of the conduction band, results in the widening of the energy gap for the mono-halide samples. Another interesting fact regarding to the mono-halide samples was the absorption cross section of the $(3: 1)$ and $(2: 1)$ molar ratio samples. Samples with additional MAI molecules in cation A site showed low absorptivity compared to the other samples where samples with less MAI molecules absorbed more photons. The fact that additional MAI in cation A site formed more secondary bonding with the $\mathrm{PbI}_{2}$ tetragonal cage might eventually (i) reduced the number of charges available to absorb photons and get excited, and (ii) reducing the internal electric field and increased the binding energy of the charges where more energy was required to excited the charges. Samples with depletion of MAI molecules show considerably high absorptivity and multi-bandgap can be observed with the second energy gap located around $2.2 \mathrm{eV}$, which was the energy gap of the $\mathrm{PbI}_{2}$ hexagonal crystal structure.

Instead of altering energy level, $\mathrm{MAPbI}_{3-\mathrm{x}} \mathrm{Br}_{\mathrm{x}}$ samples show yet another behavior in response to the halogen substitution, which was band-bending. The energy gap of the bromide-substituted samples lied right in between the energy gap value of mono-halide $\mathrm{MAPbI}_{3}$ and $\mathrm{MAPbBr}_{3}$ perovskites, illustrated in fig 2(c). When both $\mathrm{PbI}_{2}$ and $\mathrm{PbBr}_{2}$ powder were mixed with the MAI powder, both $\mathrm{MAPbI}_{3}$ and $\mathrm{MAPbBr}_{3}$ perovskite crystal phases were formed with their own respective electronic band structure. Both electronic band structure, especially valence and conduction band, underwent relocation of energy band, which was also known as band-bending, and results in an intermediate energy value. However, this was not the case for the chloride-substituted samples. Two crystalline phase, the $\mathrm{MAPbI}_{3}$ phase and $\mathrm{MAPbCl}_{3}$ phase, might also co-exist in the hybrid-halide chloridesubstituted samples. However, the energy level of the valence band for $\mathrm{MAPbCl}_{3}$ might be too low to post any effect on the valence band of the $\mathrm{MAPbI}_{3}$ phase. The valence band of the $\mathrm{MAPbI}_{3}$ perovskite was formed by the I $5 \mathrm{p}$ orbital whereas the valence band of the $\mathrm{MAPbCl}_{3}$ perovskite was formed by the $\mathrm{Cl} 3 \mathrm{p}$ orbital, where the conduction band of both perovskites were formed by the $\mathrm{Pb} 6 \mathrm{p}$ orbital. The difference in energy between I $5 \mathrm{p}$ and $\mathrm{Cl} 3 \mathrm{p}$ orbitals might be too great that most of the charges were excited from the I $5 \mathrm{p}$ orbital and not from the $\mathrm{Cl} 3 \mathrm{p}$ orbitals. Though, charges can still be excited from $\mathrm{Cl} 3 \mathrm{p}$ orbital at a longer wavelength, but the absorption can only took place at the forbidden zone between the I $5 p$ prbital and $\mathrm{Pb} 6 \mathrm{p}$ orbital. 
No sharp peaks were observed in the photoemission spectra illustrated in fig 2(d) and 2(e). This broadening peaks can be a results of degraded samples. However, sharp peaks were obtained from the XRD analysis, indicating that the samples were not degraded. Therefore, the broadening of the photoemission peaks were the only results of spin-orbit coupling effect. The spin-orbit coupling effect results in the splitting of orbitals into several small sub-orbital. With the splitted sub-orbital, electrons were relaxed to several different energy level, emitting photons with different wavelength depending to the energy level they ended up in. This phenomenon results in the broadened photoemission peak. The irregular crystal surface, results from the addition of MAI ions or substitution of ions with different ionic radii, gave rise to even more energy trap step, trapping the exciton before recombination on the valence band.

\section{Conclusion}

In conclusion, we studied the crystal structure as well as optical properties of the perovskite samples upon halide substitution and the relationship between the crystal structure and optical properties of this sample. Hybrid-halide experienced higher lattice strain compared to the mono-halide samples due to the difference in size between the substituted halide ions and the existing halide ions where substituting chloride ions generated more lattice strain compared to the bromide ions. In term of optical properties, band bending phenomenon can be observed in MAPbI3-xBrx samples when iodide ions were substituted by bromide ions. However, the energy gap of MAPbI3-xClx bends slightly when iodide ions were substituted by chloride ions, mainly due to the huge different between the $\mathrm{Cl} 3 \mathrm{p}$ orbital and I $5 \mathrm{p}$ orbital. Both spinorbit coupling and irregular surface or impurities due to the uncontrolled ambient environment gave rise to the formation of sub-orbital energy level and energy trap steps, resulting in energy loss during exciton recombination. This study aimed to provide some knowledge on providing new perovskite materials by studying the effect of ionic radii of different ions on the crystal structure and optical profile of the perovskite. The effect of halogen substitution on the full PSC device's performance need to be carried out in order to gain full understand on the halogen substitution effect.

\section{Acknowledgments}

Million thank Professor Dr. Jose Rajan for his financial and knowledge support throughout the study. This study is funded by the Material Technology Department, Faculty of Industrial Sciences \& Technology (FIST) of Universiti Malaysia Pahang. I would like to also thank Dr. Azhar Fakharuddin for all the useful discussions and assistances.

\section{References}

1. Movellan, J., The 2016 Global PV Outlook: US, Asian Markets Strengthened by Policies to Reduce CO2. 2016.

2. Kojima, A., et al., Organometal Halide Perovskites as Visible-Light Sensitizers for Photovoltaic Cells. Journal of the American Chemical Society, 2009. 131(17): p. 6050-6051.

3. Saliba, M., et al., Cesium-containing triple cation perovskite solar cells: improved stability, reproducibility and high efficiency. Energy \& Environmental Science, 2016. 9(6): p. 1989-1997. 
4. Manshor, N.A., et al., Humidity versus photo-stability of metal halide perovskite films in a polymer matrix. Physical Chemistry Chemical Physics, 2016. 18(31): p. 21629-21639.

5. Fakharuddin, A., et al., Vertical TiO2 Nanorods as a Medium for Stable and HighEfficiency Perovskite Solar Modules. ACS Nano, 2015. 9(8): p. 8420-8429.

6. Liu, D., J. Yang, and T.L. Kelly, Compact Layer Free Perovskite Solar Cells with 13.5\% Efficiency. Journal of the American Chemical Society, 2014. 136(49): p. 17116-17122.

7. Liu, M., M.B. Johnston, and H.J. Snaith, Efficient planar heterojunction perovskite solar cells by vapour deposition. Nature, 2013. 501(7467): p. 395-398.

8. Chen, Q., et al., Planar Heterojunction Perovskite Solar Cells via Vapor-Assisted Solution Process. Journal of the American Chemical Society, 2014. 136(2): p. 622625 .

9. Zhou, H., et al., Interface engineering of highly efficient perovskite solar cells. Science, 2014. 345(6196): p. 542.

10. Stranks, S.D., et al., Electron-Hole Diffusion Lengths Exceeding 1 Micrometer in an Organometal Trihalide Perovskite Absorber. Science, 2013. 342(6156): p. 341.

11. Wang, Q., et al., Large fill-factor bilayer iodine perovskite solar cells fabricated by a low-temperature solution-process. Energy \& Environmental Science, 2014. 7(7): p. 2359-2365.

12. Roldan-Carmona, C., et al., High efficiency methylammonium lead triiodide perovskite solar cells: the relevance of non-stoichiometric precursors. Energy \& Environmental Science, 2015. 8(12): p. 3550-3556.

13. Kim, J., C.-H. Chung, and K.-H. Hong, Understanding of the formation of shallow level defects from the intrinsic defects of lead tri-halide perovskites. Physical Chemistry Chemical Physics, 2016. 18(39): p. 27143-27147.

14. Mosconi, E., P. Umari, and F. De Angelis, Electronic and optical properties of MAPbX3 perovskites $(X=I, B r, C l)$ : a unified DFT and $G W$ theoretical analysis. Physical Chemistry Chemical Physics, 2016. 18(39): p. 27158-27164.

15. Carignano, M.A., et al., A close examination of the structure and dynamics of $\mathrm{HC}(\mathrm{NH} 2) 2 \mathrm{PbI} 3$ by $\mathrm{MD}$ simulations and group theory. Physical Chemistry Chemical Physics, 2016. 18(39): p. 27109-27118.

16. He, Y. and G. Galli, Instability and Efficiency of Mixed Halide Perovskites CH3NH3AI3-xClx $(A=P b$ and Sn): A First-Principles, Computational Study. Chemistry of Materials, 2017. 29(2): p. 682-689. 